$\operatorname{CONF} \mathscr{E} 1114-25$

CONF-861114--25

DE87 002402

\title{
CALCULATIONS OF COINCIDENT IONIZATION PLUS EXCITATION*
}

\author{
Richard L. Becker \\ Oak Ridge National Laboratory \\ Oak Ridge, TN 37831-6373, USA
}

For Li- and Be-like ions, $K \times$-ray yields, together with detection that the ionic charge has increased, give the cross section for ionization plus excitation (IE), a process which can exhibit electron-electron correlations. Measurements of IE for ${ }_{14} \mathrm{Si}^{11+}+$ He stimulated our coupled-channels calculations in the independent-Fermi-particle model (IFPM), which includes Pauli correlations. We discuss how the IFPM expressions, generalized here to include an open shell, differ from those for distinguishable electrons. The sensitivity of $\sigma_{I E}$ to correlations is shown. Recent additional measurements and future ones giving excitation functions for resolved configurations and complementary Auger data will provide even more sensitive tests of collisional correlation theory.

\section{DISCLAIMER}

\begin{abstract}
This report was prepared as an account of work sponsored by an agency of the United States Government. Neither the United States Government nor any agency thereof, nor any of their employees, makes any warranty, express or implied, or assumes any legal liability or responsibility for the accuracy, completeness, or usefulness of any information, apparatus, product, or process disclosed, or represents that its use would not infringe privately owned rights. Reference herein to any specific commercial product, process, or service by trade name, trademark, manufacturer, or otherwise does not necessarily constitute or imply its endorsement, recommendation, or favoring by the United States Government or any agency thereof. The views and opinions of authors expressed herein do not necessarily state or reflect those of the United States Government or any agency thereof.
\end{abstract}

* Research sponsored by the Division of Chemical Sciences, Office of Basic Energy Sciences, U.S. Department of Energy under contract DE-AC05-840R21400 with Martin Marietta Energy Systems, Inc. 
1. Introduction

In the last few years experiments on ion-atom collisions have been made in which projectile $x$-rays are detected in coincidence with the final charge-state of the ion. When the ionic charge is decreased the process, originally called electron capture with excitation (CE) [1], is now referred to most often as electron transfer (to the projectile) and excitation (TE) [2]. When the charge is increased the process may be called electron loss [2] with excitation (LE) or ionization plus excitation (IE). Both TE and IE require at least two electrons to make transitions during the collision, in contrast to non-charge-changing $x$-ray producing events. The TE process can occur by two successive interactions of the nuclear charges (NTE, $N=$ "non-resonant") or by the electron-electron interaction (RTE, $R=$ "resonant") [3]. The similarity of RTE to dielectronic recombination has been stressed [1-4], and the quantal interference between NTE and RTE has been treated [5]. The wide RTE peaks are related to the narrow Auger resonances by vast kinematic broadening $\left(\propto m_{p} / m_{e}\right)$ and by the momentum distribution (Compton profile) of the target electron which is captured. Well-separated contributions from NTE and RTE in the same collision system have been seen [6].

In IE there is no necessity for any target electron to come close to either the projectile electron being ejected or the one being excited. The IE process is calculated here without inclusion of the interactions between projectile and target electrons. My interest in IE stems from two kinds of "correlations" which it can exhibit. First, like any two-electron reaction, the cross-section may not be a simple function of the cross sections for the component single-electron reactions, i.e. there may be a "correla- 
tion" between the excitation and the ionization processes not inferrable from the integrated singles cross sections. In the semi-classical, impactparameter treatment we use, even if the IE expected value $Q^{I E}(b)$ iactors into

$$
Q^{I E}(b)=Q^{I}(b) \cdot Q^{E}(b) \text {, }
$$

the cross section

$$
\sigma^{I E}=2 \pi \int_{0}^{\infty} d b \cdot b Q^{I E}(b)
$$

may not be related simply to $\sigma^{I}$ and $\sigma^{E}$, and the ratio $\sigma^{I} \cdot \sigma^{E} / \sigma^{I E} \equiv \pi R^{2}$ may vary with $E / A, Z_{p}$, and $Z_{T}$. Second, there may be correlations, at a given value of the impact parameter, between the two active electrons. More preciseily, the transition probability for the two-electron process may not factor into single-electron transition probabilities, so that Eq. (1a) will not hold.

IE data have been reported for Li-like $S^{1+}+\mathrm{Ar}[2], \mathrm{Si}^{11+}+\mathrm{He}[7]$, $\mathrm{Si}^{1+}+\left(\mathrm{Ne}\right.$ and $\mathrm{Ar}$ ) [8], $\mathrm{Ca}^{17+}+\mathrm{H}_{2}$ [9], and for He-, Li-, and Be-like $\mathrm{Ca}^{q^{+}}+\mathrm{He}$ [10]. In ref. [9] there is shown a fascinating anti-correlation between the IE and TE $K_{\alpha \beta}$ cross sections which, to my knowledge, is completely unexplained and will not be discussed here.

We have developed a theoretical treatment of IE which involves the following approximations: it is non-relativistic, treats the relative motion of the ion and atom classically, neglects the possibility of the flipping of intrinsic spins by the spin-orbit interaction, employs the independent fermi particle model (IFPM) [11], and allows for the electrons on the neutral target atom only through the use of a screened effective nuclear charge. The last approximation may be too crude. One could use an impact-parameter-dependent nuclear charge, but we have not yet done so. 
For a He target we did a preliminary calculation for a bare nucleus with $Z=11$ (simulating $\mathrm{Si}^{1 \mathrm{l}+}$ ) on $\mathrm{He}$ at $50 \mathrm{MeV}(\mathrm{E} / \mathrm{A} \cong 1.8 \mathrm{MeV})$. The probability of ionizing each He 1s electron was 0.99 for $b=0$ and fell below 0.7 only for $b$ greater than twice the $\mathrm{K}$-shell radius of $\mathrm{He}$, i.e. greater than roughly 14 times the $\mathrm{K}$-shell radius of $\mathrm{Si}$. Thus, we think He presents an effective $Z=2$ to $\mathrm{Si}^{1 \mathrm{l}+}$ for the purposes of calculating IE.

In our model the two-electron transition probabilities do not factor only if the intrinsic spins have the same projection $\left(m_{s}\right)$. Thus, Li-like projectiles are the simplest which can exhibit Pauli exclusion terms (PETs). For this reason, and because the 3-electron system provides the simplest opportunity of generalizing our previous work on the IFPM to include a partially open shell, we have begun our work on IE with a study of Li-like projectiles, in particular Sillt, for which data are available for $K_{\alpha}$ and $K_{B} x$-rays in collisions with $\mathrm{He}, \mathrm{Ne}$, and $\mathrm{Ar}$. He- and Be-like projectiles will require relatively small modifications to our computer programs. Because we exclude the target electrons from playing an active role, we interchange the roles of projectile and target and treat the case of $Z_{p}+S i^{11+}$ collisions, where $Z_{p}$ stands for a bare nuclear projectile with effective nuclear charge $Z_{p}$. Then we can do coupled-channels calculations with the program of ref. 12, which employs a target-centered expansion in Hartree-Fock bound states and pseudostates in the continuum.

\section{IFPM formalisin for Li-like $\left(1 s^{2} 2 s\right)$ projectiles}

The direction of the intrinsic spin of the 2 s electron will be up (t). Then only the up-pair $(1 s+2 s+)$ will exhibit exchange terms in the initial -Hartree-Fock ground state and in the transition amplitudes. For the uppair the probability of occupying the states $k$ and $k^{-}$after the collision 
is given in terms of single-electron transition amplitudes $a_{k, k}$ - by [11]

$$
p^{k, k^{\prime}}=p^{k} p^{k^{-}}-\left|a_{k, k}^{(2)}-\right|^{2} \text {, }
$$

where the inclusive single-particle occupancy probability for spin-orbital $k$ is

$$
p^{k}=\left|a_{k, 1 s}\right|^{2}+\left|a_{k, 2 s}\right|^{2}
$$

and the PET amplitude is

$$
a_{k, k^{\prime}}^{(2)}=\sum_{h=1 s, 2 s} a_{k, h^{*} k^{*}, h^{*}}
$$

There are partial cancellations (Paul i blocking) leading to

$$
\begin{aligned}
& p^{k, k^{\prime}}=\left|a_{k, 1 s}\right|^{2}\left|a_{k^{\prime}, 2 s}\right|^{2}+\left|a_{k, 2 s}\right|^{2}\left|a_{k^{\prime}, 1 s}\right|^{2}
\end{aligned}
$$

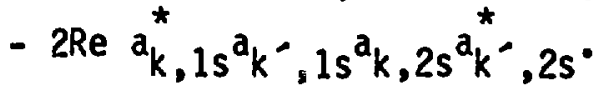

The probability that both up-electrons remain bound is

$$
p^{B, B}=\sum_{b, b^{-}}^{B N D} p^{b, b^{-}} \quad\left(b<b^{-}\right) .
$$

The inclusive probabilities that the electron initially in the occupied state $h$ is either (1) ionized or (2) excited into the $2 p$ shell are

$$
\begin{aligned}
& p^{I}(h)=\sum_{c}^{\text {UNBND }}\left|a_{c, h}\right|^{2}=1-\sum_{b}^{B N D}\left|a_{b, h}\right|^{2}=1-p^{B}(h), \\
& p^{2 p}(h)=\sum_{m_{l}}\left|a_{2 p m_{l}, h}\right|^{2} .
\end{aligned}
$$

With these ingredients the expected value for inclusive single ionization (including double and triple ionization) is

$$
Q^{I}=1 \cdot\left(1-P^{B, B}\right)+P^{I}(1 s t) \cdot P^{B, B}
$$

Here $1-p^{B, B}$ is the expected value that one or both of the up-electrons is ionized, and the factor 1 in front of $\left(1-p^{B, B}\right)$ is a reminder that the downelectron either may be ionized or remain bound. The inclusive expected value for forming a $k_{\alpha}$ configuration, $2 p m_{s}\left(1 s m_{s}\right)^{-1}$, irrespective of the 
charge state $q$ (i.e., including $z-q=3,2$, and 1 ) is

$$
\begin{aligned}
& Q\left(k_{\alpha}\right)=\sum_{m_{s}} P_{1 s m_{s}}^{2 p m_{s}}=p^{2 p \downarrow}(1 s t) \cdot 1+1 \cdot\left\{p^{2 p \uparrow}(1 s+)\left[1-\left|a_{1 s t, 2 s \uparrow}\right|^{2}\right]+\right. \\
& \left.+\left[1-\left|a_{1 s \uparrow, 1 s+}\right|^{2}\right] p^{2 p \dagger}(2 s \uparrow)+2 \operatorname{Re} \sum_{m_{\ell}} a_{2 p m_{\ell}, 1 s^{a} 1 s, 1 s^{a} 2 p m_{\ell}, 2 s^{a} 1 s, 2 s}^{\star}\right\} \text {. }
\end{aligned}
$$

We have worked out IFPM expressions for the expected values of $k_{\alpha}$ configurations in ions with a specific final ionic charge, i.e. Z-q exclusively 3,2, or 1 . Their sum is the inclusive quantity $Q\left(k_{\alpha}\right)$ given above. Data are available for the single-ionization coincidence with $K_{\alpha}$. Our expression for this is

$$
\begin{aligned}
Q^{I}\left(K_{\alpha}\right) & =p^{I}(1 s t) \cdot\left[p_{1 s}^{2 p, B}+p^{\left.2 p_{0}, 2 p_{1}^{+}\right]}+p^{2 p}(1 s t) \cdot p^{B, I}+\right. \\
& +p^{B}(1 s t) \cdot\left[p^{2 p}(1 s)+p^{2 p}(2 s)-p^{2 p, B}-p^{2 p_{0}, 2 p_{1}^{+}}\right]
\end{aligned}
$$

Here $P_{1 s}^{2 p, B}$ is the probability that one up-electron is in a $2 p$ orbital while the other is bound but not in the 1s (subscripts label vacancies [11]). The $2 p\left(m_{\ell}=1\right)$ states can be separated into a combination even under time reversal $\left(2 p_{1}^{+}\right)$, which is excitable from an s-state, and an odd one which is not. The configuration $\left(2 p_{0}, 2 p_{1}^{+}\right)$is counted twice because it has two ways of producing a $k_{\alpha} x$-ray. $p^{B, I}$ is the probability that one up-electron is bound and the other is ionized. All the correlated quantities for two upelectrons involve interference terms. In order to separate $Q^{I^{1}}\left(K_{\alpha}\right)$ into parts with $2 s+2 p$ and $1 s+2 p$ we assign half the values of the interference terms in $p^{2 p, B}, p^{2 p_{0} 2 p_{1}^{+}}$, and $p_{1 s}^{2 p, B}$ to each part, with the caveat that interferring processes cannot truly be separated. Expressions for the production of $K_{B}$ configurations are obtained from the ones above simply by replacing $2 p$ by $3 p$ everywhere. 
3. Results of IFPM-coupled-channels calculations for $\mathrm{Sillt}$

First we give results of calculations of cross sections for $K_{\alpha}$ and $K_{B}$ configurations coincident with the ejection of 0,1 , or 2 electrons at a relatively high impact energy where the inclusive $x$-ray cross sections are large. In Fig. 1 one sees that for $Z p=2$ almost all the $K \times$-rays come from $\mathrm{Si}^{1{ }^{1+}}$; the $\mathrm{K}-\mathrm{Si} \mathrm{IL}^{12+}$ component is only $\sim 5 \%$ as large. However, as $Z_{p}$ increases, for small $Z_{p}$ each ejected electron gives an extra factor of $Z_{p}{ }^{2}$ so that at $z_{p}=8$ or $9, \sigma^{I^{1}}(K) \sim \sigma^{I^{0}}(K)$, and for $z_{p} \approx 14$ or $15, \sigma^{I^{2}}(K) *$ $\sigma^{I^{0}}(K)$. The unitary nature of the coupled-channels theory guarantees that these cross sections saturate as a function of $Z_{p}$. This is quite apparent in the behavior of $\sigma^{I^{0}}(K)$. For Ne and Ar targets it would be quite valuable to have separate data for all three charge states and not merely the $K$ $x$-ray singles and $\left(K x-r a y, S i^{12+}\right)$ coincidence data.

Data for $K_{B} x$-rays are rather scarce so far, but the UNC group reports [15] that, for $S i^{11+}+$ He with $E / A$ between 2 ana $3 \mathrm{MeV}$, the average $\sigma_{K_{B}} / \sigma_{K_{\alpha \beta}}$ is -0.17 , in good agreement with our 0.152 , and the average $\sigma_{K_{p}}^{12+} / \sigma_{K_{\alpha \beta}}^{12+}$ is 0.21 to be compared with our 0.097. Preliminacy analysis [10] for $\mathrm{Ca} 17++\mathrm{He}$ with $\mathrm{E} / \mathrm{A} \cong 3$ to $8 \mathrm{MeV}$ gives $\sigma_{K_{\beta}} / \sigma_{K_{\alpha \beta}} \cong 0.1$ to 0.2 and $\sigma_{K_{\beta}}^{18+} / \sigma_{K_{\alpha \beta}}^{18+}$ $=0.02$ to 0.05 . For heavier collision partners Fig. 1 gives $\sigma_{K_{\beta}}^{12+} / \sigma_{K_{\alpha \beta}}^{12+}=$ $=0.107$ for $Z_{p}=10$ and 0.182 for $Z_{p}=18$.

Next we turn to calculations for the $\mathrm{Si}^{11+}+\mathrm{He} \mathrm{K}_{\alpha}$ experiments [7] as a function of impact energy per nucleon, $E / A$, from 0.5 to $3.5 \mathrm{MeV}$. Figure $2 a$ shows that the inclusive single ionization is fairly well reproduced by the calculation. For the inclusive $k_{\alpha}$ cross section, Fig. $2 b$, the upper "theoretical curve falls about $10 \%$ to $25 \%$ below the data. However, the mean branching ratio (fluorescence yield, $(\omega)$ was taken to be unity, whereas we 
expect $w=0.5$ both from experiment [13] and from theory [14]. For the $K_{\alpha}{ }^{-S} i^{12+}$ coincidence, we show separately in Fig. $2 c$ the contribution of $1 s t+2 p t$ in Eq. (11), the remainder which involves excitation of $2 p \uparrow$ from either the $1 \mathrm{~s} \uparrow$ or the $2 \mathrm{~s} \uparrow$ or an interference between them, and their sum. Then in Fig. 3 two alternative decompositions are given. The important role of the interference terms is shown. The dominance of the $1 s+2 p$ is even more striking for $K_{B}-S i 12+$ in He, but is greatly reduced as $Z_{p}$ increases (Table 1). Resolution of the factor of 2 discrepancy between calculated and experimental $x$-ray yields remains an important task. Auger data would help, as would data for resolved configurations.

I thank S. Shafroth, J. Anthony, J. Swenson, J. Tanis, and E. Bernstein for interesting me in this area of collision phenomena, for discussions, and for sending me data before publication; and $Y$. Hahn for his calculated fluorescence yields. 


\section{References}

[1] J. A. Tanis, S. M. Shafroth, J. E. Willis, M. Clark, J. Swenson, E. N. Strait, and J. R. Mowat, Phys. Rev. Lett. 47 (1981) 829.

[2] J. A. Tanis, E. M. Bersntein, W. G. Graham, M. Clark, S. M. Shafroth, B. M. Johnson, K. W. Jones, and M. Meron, Phys. Rev. Lett. 49 (1982) 1325.

[3] D. Brandt, Phys. Rev. A27 (1983) 1314.

[4] J. A. Tanis, E. M. Bernstein, W. G. Graham, M. P. Stock1i, M. Clark, R. H. McFarland, T. J. Morgan, K. H. Berkner, A. S. Schlacter, and J. W. Stearns, Phys. Rev. Lett. 53 (1984) 2551.

[5] J. M. Feagin, J. S. Briggs, and T. M. Reeves, J. Phys. B17 (1984) 1057.

[6] M. Clark, D. Brandt, J. K. Swenson, and S. M. Shafroth, Phys. Rev. Lett. 54 (1985) 544.

[7] M. Clark, J. Anthony, J. K. Swenson, and S. M. Shafroth, in: Electronic and Atomic Collision, Abstracts of Contributed Papers, eds., M. J. Coggiola, D. L. Huestis, and R. P. Saxon (14th ICPEAC, Palo Alto, 1985) p. 398.

[8] S. M. Shafroth, in: Progress Report for Cc tract DE-AS05-78ER05027 between the University of North Carolina, Chapel Hill, and the U.S. Department of Energy, Aug. 1984-July 1985, pp. 23-24.

[9] E. M. Bernstein, J. A. Tanis, K. H. Berkner, M. W. Clark, W. G. Graham, R. H. McFarland, T. J. Morgan, A. Müller, A. S. Schlachter, and J. W. Stearns, 14th ICPEAC, loc. cit., p. 395. 
[10] W. G. Graham, E. M. Bernstein, M. W. Clark, C. S. Oglesby, J. A. Tanis, K. H. Berkner, P. Gohil, A. S. Schlachter, J. W. Stearns, B. M. Johnson, K. W. Jones, M. Meron, R. H. McFarland, T. J. Morgan, A. Müller, and M. P. Stockli, in: Proc. 2nd U.S.-Mexico Coop. Symp. on Atomic \& Molec. Phys.: Two-Electron Phenomena, Cocoyoc, Mexico, Jan. 1986, to be published.

[11] R. L. Becker, A. L. Ford, and U. F. Reading, Phys. Rev. A29 (1984) 3111.

[12] M. H. Martir, A. L. Ford, J. F. Reading, and R. L. Becker, J. Phys. B15 (1982) 2405.

[13] B. L. Doyle, U. Schiebel, J. R. Macdonald, and L. D. Ellsworth, Phys. Rev. A17 (1978) 523.

[14] I. Nasser and Y. Hahn, J. Quant. Spectrosc. Radiat. Transfer 29 (1983) 1; Y. Hahn, priv. comm.

[15] J. M. Anthony and S. M. Shafroth, priv. comm. 


\section{Table 1}

Contributions of (1) $1 s+2 p$ or $3 p, 2 s$ or $1 s+I\left(\sigma_{1}\right)$, and (2) $2 s+2 p$ or $3 p$, $1 s+1\left(\sigma_{2}\right)$ to $\sigma_{K_{\alpha}}^{12+}$ or $\sigma_{K_{B}}^{12+}$ (in units of $10^{-20} \mathrm{~cm}^{2}$ ) in $2 p+S i 11+$ collisions at $E / A=3.2143 \mathrm{MeV}\left(90^{\mathrm{MeV}}{ }^{28} \mathrm{Si}\right)$.

\begin{tabular}{|c|c|c|c|}
\hline$Z_{p}$ & $\sigma_{1}$ & $\sigma_{2}$ & $\sigma_{1} / \sigma_{2}$ \\
\hline $\begin{array}{r}2 \\
10 \\
18\end{array}$ & $\begin{array}{l}5.42 \times 10^{-2} \\
11.0 \\
13.2\end{array}$ & $\begin{array}{l}3.46 \times 10^{-2} \\
16.2 \\
44.3\end{array}$ & $\begin{array}{l}1.57 \\
0.68 \\
0.30\end{array}$ \\
\hline $\begin{array}{r}2 \\
20 \\
18\end{array}$ & $\begin{array}{l}8.26 \times 10^{-3} \\
1.74 \\
3.11\end{array}$ & $\begin{array}{l}1.27 \times 10^{-3} \\
1.53 \\
9.70\end{array}$ & $\begin{array}{l}6.50 \\
1.14 \\
0.32\end{array}$ \\
\hline
\end{tabular}




\section{Figure Captions}

Fig. 1. Calculated cross sections in units of $10^{-20} \mathrm{~cm}^{2}$ for $K_{\alpha}$ and $K_{\beta}$ configurations $(\bar{\omega}=1)$ in coincidence with $\mathrm{Si}^{11+}, \mathrm{Si}^{12+}$, or $\mathrm{Si}^{13+}$ in $Z_{p}+S i i+\left(1 s^{2} 2 s\right)$ collisions at $E / A=3,2143 \mathrm{MeV}(90 \mathrm{MeV} 28 \mathrm{Si})$ versus the effective nuclear charge, $z_{p}$, of the collision partner. The curves are labeled by 0 (dash), 1 (dash-dot), and 2 (dashdouble dot), the number of electrons ejected. The full curve is the inclusive $K$-configuration cross section not including electron capture events, equal to the sum of the cross sections labeled 0 , 1 , and 2.

Fig. 2. Experimental $[7,8]$ and theoretical cross sections for $\mathrm{Si}^{1++}+\mathrm{He}$ versus impact energy per amu. a) Inclusive single ionization. The full curve is direct ionization; the dashed one includes excitation-autoionization assuming $\bar{\omega}=0$, b) Inclusive $K_{\alpha} x$-ray emissiun from Si ions with $\Delta q>0$. The data are the fully inclusive ones minus those for TE $[6]$. The lower curve lacks the interference term, the upper one includes it. c) Exclusive $K_{\alpha}-S i^{12+}$ coincidence in units of $10^{-21} \mathrm{~cm}^{2}$. The lowest curve (dot) is the contribution involving $15 t+2 p t$, the middle curve (dash) involves (1s or $2 s) \uparrow+2 p \uparrow$, and the top curve (full) is their sum.

Fig. 3. Two decompositions of the theoretical $K_{\alpha}-S i 12+$ cross section for $\mathrm{He}^{2+}+\mathrm{Si}^{11+}$. First, a separation into two direct terms (lower full and long-dashed) and their interference (short-dashed). The 
approximate significance of the direct terms is: lower full, $1 s+2 p$ and mostly $2 s$ ionized; lower dashed, $1 s$ ionized and $2 p$ excited mostly from the $2 s$. Second, a different separation into excitation of the $2 p$ from 1 s (upper full) and from $2 s$ (upper dashed), each including one half the interference between these processes. 


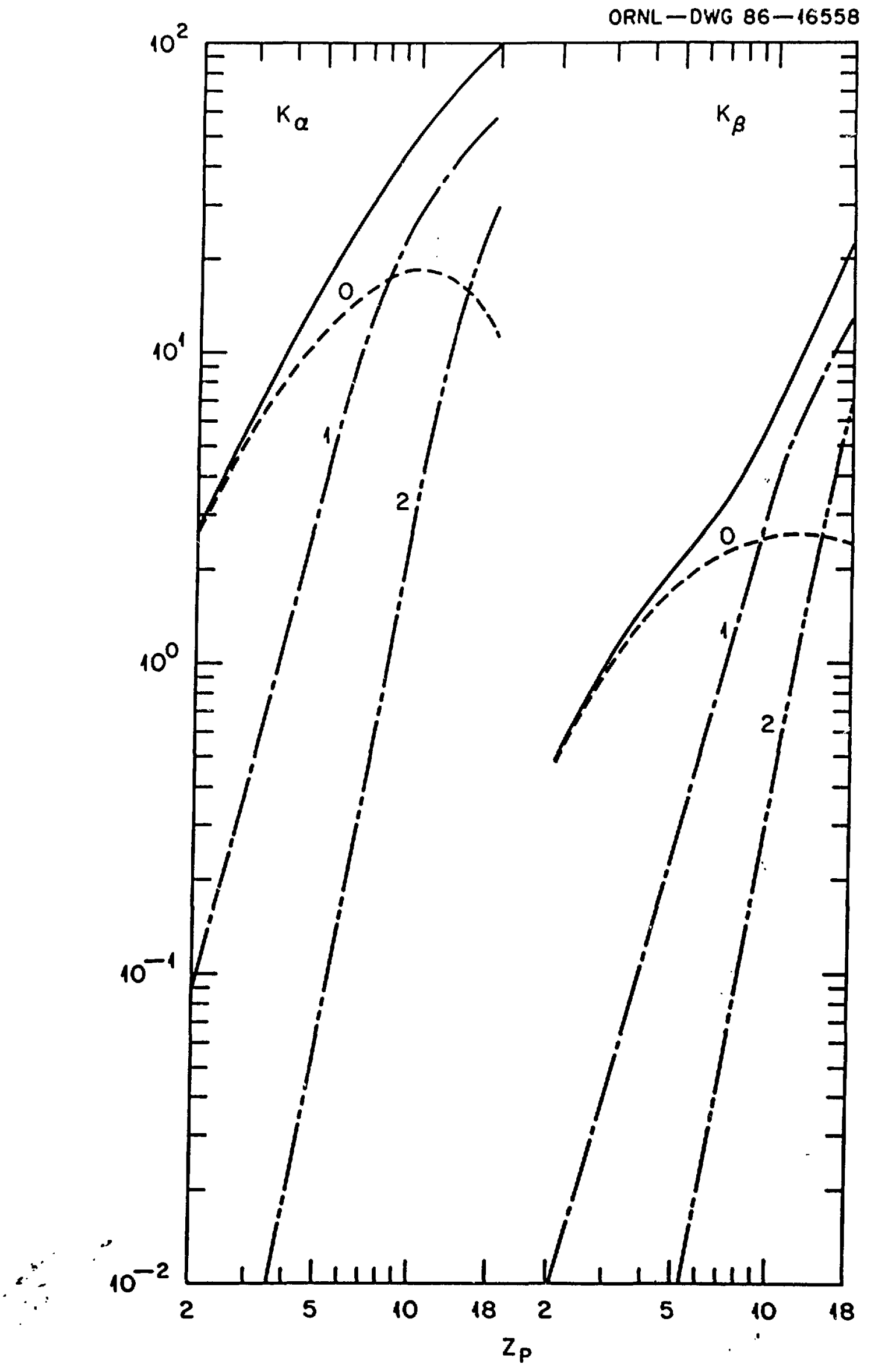

Fia. 1 


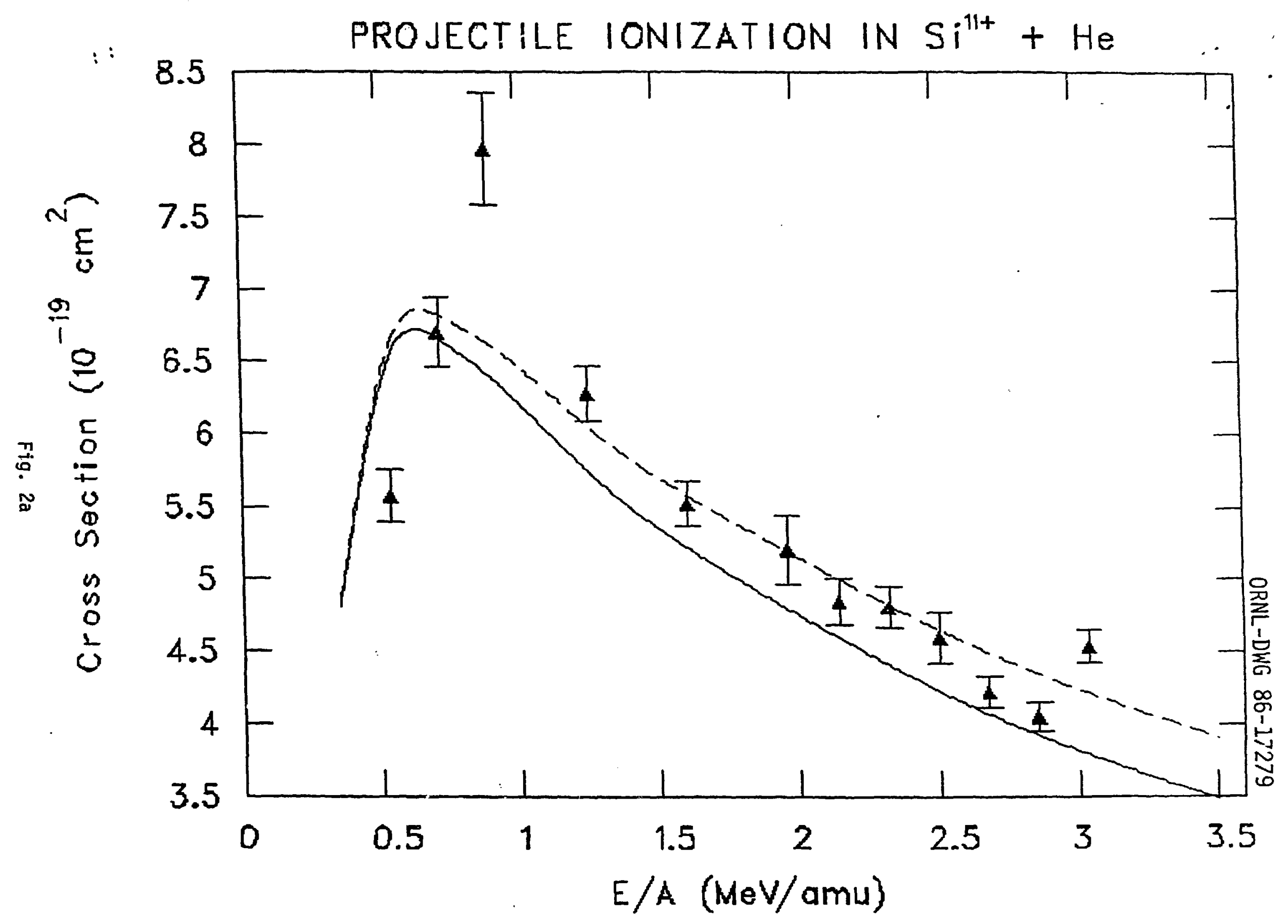




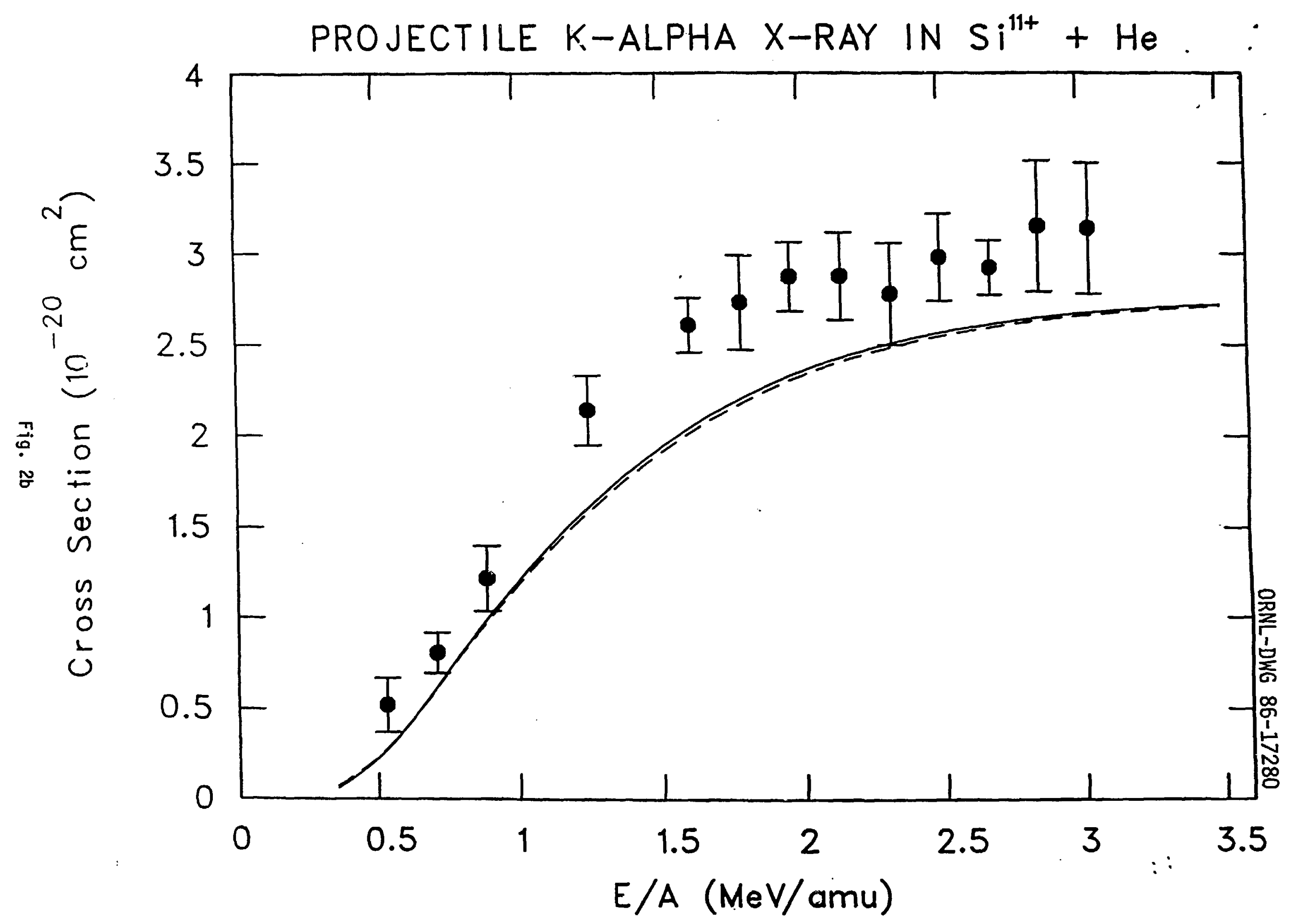




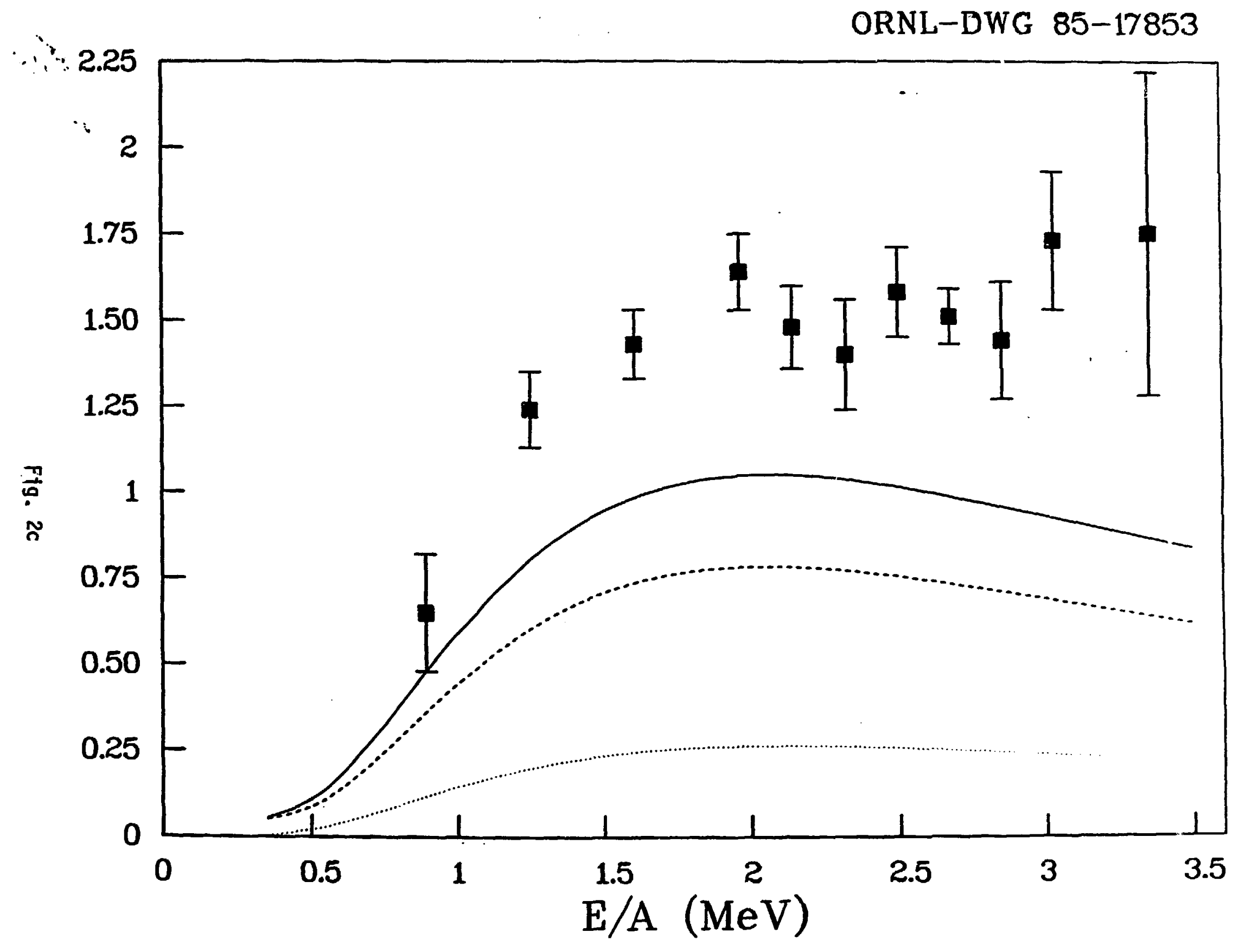




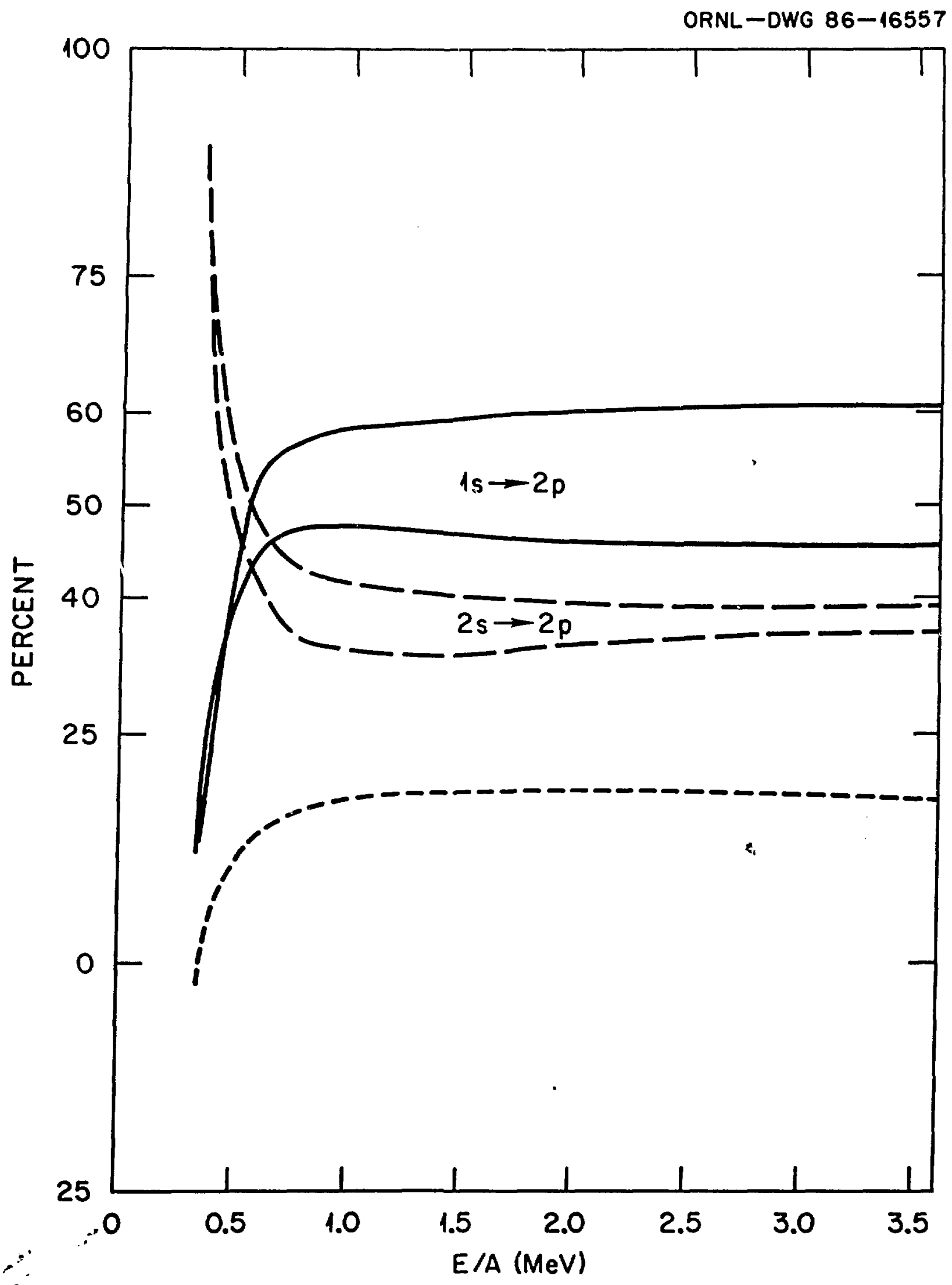

Fig. 3 